\title{
Relaxation of Energy and Momentum in an Carrier-Phonon System
}

\author{
A. Rossani \\ Department of Applied Science and Technology, Politecnico di Torino, Corso Duca degli Abruzzi, Torino, Italy \\ Email: alberto.rossani@polito.it
}

Received June 25, 2012; revised July 19, 2012; accepted August 1, 2012

\begin{abstract}
If electrons $(e)$ and holes $(h)$ in metals or semiconductors are heated to the temperatures $T e$ and $T h$ greater than the lattice temperature $T p$, the electron-phonon interaction causes energy relaxation. In the non-uniform case a momentum relaxation occurs as well. In view of such an application, a new model, based on an asymptotic procedure for solving the generalized kinetic equations of carriers and phonons is proposed, which gives naturally the displaced Maxwellian at the leading order. After that, balance equations for the electron number, hole number, energy densities, and momentum densities are constructed, which constitute now a system of five equations for the electron chemical potential, the temperatures and the drift velocities. In the drift-diffusion approximation the constitutive laws are derived and the Onsager relations recovered.
\end{abstract}

Keywords: Energy; Momentum

\section{Introduction}

In semiconductor modeling three approaches are widely applied, according to the Physical situation we deal with. The microscopic approach is based on Monte Carlo simulations which can account for as many aspects of semiconductor Physics as we want. Nevertheless it is well known that these simulations are time consuming and therefore expensive.

The mesoscopic approach is based on the Boltzmann transport equation (BTE). Several numerical techniques are developed in order to face a numerical solution of the problem. The distribution function depends on seven variables (momentum, position, time) so that the task is quite formidable.

The macroscopic approaches are based on the weak form of the BTE, which give rise to a hierarchy of coupled equations for the moments of the distribution function. Such approach requires a truncation at some order based on closure assumptions for the higher order fluxes and for the production terms for non-conservation equations.

Most of the semiconductor macroscopic models have in common the assumption, at the basis of the closure approximation, that some higher moments can be calculated by utilizing a displaced Maxwellian. This approach would be justified if one had a systematic approximation for solving the Boltzmann transport equations, asymptotic with respect to some parameters, whose leading terms would be displaced Maxwellians. Such an approach is now available and the present model can be viewed as an improvement of a previous paper [1] on this topic, by considering a two-fluid description of the electron-phonon system which occurs.

On the other hand, as pointed out in ref. [2], fractal or power law distribution functions are of interest in solid state Physics. An example, given in ref. [2], is the thermalization, due to the electron-phonon interactions, of a non-equilibrium electron-phonon system which occurs if electrons and holes in metals or semiconductors are heated to a temperature $T_{e}$ greater than the lattice temperature $T_{p}$ In the non-uniform case a momentum relaxation occurs as well.

In view of such an application we consider a generalized kinetic theory of carriers and phonons (GKTCP), recently proposed [3], which accounts for non-Gibbsian statistics.

We start from the generalized Bloch-BoltzmannPeierls coupled equations for the distribution functions of electrons and phonons.

After that, by means of an expansion of both the unknowns and the interaction kernels with respect to a small parameter which accounts for the umklapp processes (with no momentum conservation), the lowest order equations show that the displaced Maxwellian approximation is justified. A closed set of two-fluid equations for the chemical potential of electrons, the temperatures, and the drift velocities can be constructed, which recalls 
the extended thermodynamics model [4].

In the drift-diffusion approximation the constitutive equations can be written and the Onsager symmetry relations are recovered.

We stress that in the present model

1) The displaced Maxwellian approximation is not an ad hoc assumption but is justified by the expansion we apply.

2) The statistics of both electrons, holes and phonons is left general, in order to account for non-standard cases.

3) Phonons are treated as a partecipating species, which brings energy and momentum.

4) The correct phonon-phonon, electron-phonon, electron-electron, hole-phonon, and hole-hole interaction kernels are utilized: we avoid the use of relaxation time approximations.

The most qualifying point is (3). In fact the usual assumption that the phonon field can be treated as a fixed background is dropped here, since "any thermal gradient give rise to transport of heat by the phonons, whilst an electric current, though carried by electrons, cannot fail to transfer some of its momentum to the lattice vibrations, and drag them along with it" (Ziman). Finally we can say that the present model can be seen as generalization of previous ones like [5], by means of the treatment of phonons.

\section{The GKTCP Equations}

Consider three interacting populations: electrons $(e)$, with charge- $e$, holes $(h)$, with charge $e$, and phonons $(p)$. Let $N_{g}(k, x, t)$ be the distribution function of phonons (quasimomentum $k$, energy $\omega_{g}(k)$ ) of type $g$ (i.e. branch $g$ of the phonon spectrum) $n_{\mathbf{P}}^{e}=n_{\mathbf{P}}^{e}(\mathbf{P}, x, t)$ the distribution function of electrons (quasi-momentum $\mathrm{P}$, energy $\varepsilon_{\mathbf{P}}$ ), and $n_{\mathbf{P}}^{h}=n_{\mathbf{P}}^{h}(\mathbf{P}, x, t)$ the distribution function of holes (quasi-

$$
\begin{aligned}
&\left(\frac{\partial N_{g}}{\partial t}\right)_{p p} \\
& \int\left[( \frac { 1 } { 2 } ) \sum _ { g 1 g 2 } \omega _ { p p } ( \mathbf { k } _ { 1 } , \mathbf { k } _ { 2 } \rightarrow \mathbf { k } ) \left(-\Phi_{g} \Psi_{g 1}\right.\right. \\
& \mathbf{k}_{2}=\mathbf{k}-\mathbf{k}_{1}+\mathbf{b}\left(\mathbf{k}_{1}, \mathbf{k}_{2} \rightarrow \mathbf{k}\right), \\
& \mathbf{k}_{3}=\mathbf{k}+\mathbf{k}_{1}+\mathbf{b}\left(\mathbf{k}, \mathbf{k}_{1} \rightarrow \mathbf{k}_{3}\right)
\end{aligned}
$$$$
=\int\left[\left(\frac{1}{2}\right) \sum_{g 1 g 2} \omega_{p p}\left(\mathbf{k}_{1}, \mathbf{k}_{2} \rightarrow \mathbf{k}\right)\left(-\Phi_{g} \Psi_{g 1} \Psi_{g 2}+\Psi_{g} \Phi_{g 1} \Phi_{g 2}\right)+\sum_{g 1 g 3} \omega_{p p}\left(\mathbf{k}_{1}, \mathbf{k}_{2} \rightarrow \mathbf{k}_{3}\right)\left(\Psi_{g} \Psi_{g 1} \Phi_{g 3}-\Phi_{g} \Phi_{g 1} \Psi_{g 3}\right)\right] \frac{\mathrm{d} \mathbf{k}_{1}}{8 \pi^{3}}
$$

where

(b is an appropriate vector belonging to the reciprocal lattice), which account for three phonon processes:

$$
\begin{array}{cc}
(g, \mathbf{k}) \rightleftharpoons\left(g_{1}, \mathbf{k}_{1}\right)+\left(g_{2}, \mathbf{k}_{2}\right), & \begin{array}{l}
\text { any quasimomenta } \mathbf{P} \text { and the number of phonons ab- } \\
\text { sorbed by electrons with any } \mathbf{P}^{\prime} .
\end{array} \\
\left(g_{3}, \mathbf{k}_{3}\right) \rightleftharpoons(g, \mathbf{k})+\left(g_{1}, \mathbf{k}_{1}\right) . & \text { For electrons we have } \\
\left(\frac{\partial n_{\mathbf{P}}}{\partial t}\right)_{e p}=\sum_{g} \int\left[\omega_{e p}\left(\mathbf{P}^{\prime}, \mathbf{k} \rightarrow \mathbf{P}\right)\left(\varphi_{\mathbf{P}^{\prime}}^{e} \psi_{\mathbf{P}}^{e} \Phi_{g}-\psi_{\mathbf{P}^{\prime}}^{e} \varphi_{\mathbf{P}}^{e} \Psi_{g}\right)+\omega_{e p}\left(\mathbf{P}^{\prime \prime} \rightarrow \mathbf{P}, \mathbf{k}\right)\left(\varphi_{\mathbf{P}^{\prime \prime}}^{e} \psi_{\mathbf{P}}^{e} \Psi_{g}-\varphi_{\mathbf{P}}^{e} \psi_{\mathbf{P}^{\prime \prime}}^{e} \Phi_{g}\right)\right] \frac{\mathrm{d} \mathbf{k}}{8 \pi^{3}}
\end{array}
$$

momentum P, energy $\varepsilon_{\mathbf{P}}^{h}$. The GKTCP equations read

$$
\begin{aligned}
& \mathcal{D}_{g} N_{g}=\left(\frac{\partial N_{g}}{\partial t}\right)_{p p}+\left(\frac{\partial N_{g}}{\partial t}\right)_{p e}+\left(\frac{\partial N_{g}}{\partial t}\right)_{p h} \\
& \mathcal{D}_{\mathbf{P}}^{\alpha} n_{\mathbf{p}}=\left(\frac{\partial n_{\mathbf{p}}}{\partial t}\right)_{\alpha p}+\left(\frac{\partial n_{\mathbf{p}}}{\partial t}\right)_{\alpha \alpha},
\end{aligned}
$$

$\alpha=e, h$ where

with

$$
\begin{gathered}
\mathcal{D}_{g}=\frac{\partial}{\partial t}+u_{g} \cdot \frac{\partial}{\partial x} \\
\mathcal{D}_{\mathbf{P}}^{e}=\frac{\partial}{\partial t}+\mathbf{v}_{e} \cdot \frac{\partial}{\partial x}-e \mathbf{E} \cdot \frac{\partial}{\partial \mathbf{P}} \\
\mathcal{D}_{\mathbf{P}}^{h}=\frac{\partial}{\partial t}+\mathbf{v}_{h} \cdot \frac{\partial}{\partial x}+e \mathbf{E} \cdot \frac{\partial}{\partial \mathbf{P}} \\
\mathbf{u}_{g}=\frac{\partial \omega_{g}}{\partial \mathbf{k}}, \mathbf{v}^{\alpha}=\frac{\partial \varepsilon_{\mathbf{P}}^{\alpha}}{\partial \mathbf{P}}, \mathbf{E}=-\frac{\partial \mathcal{P}}{\partial x},
\end{gathered}
$$

where $\mathcal{D}$ is the electric potential. Observe that, since $\omega_{g}$ and $\varepsilon_{\mathbf{p}}$ are even, $\mathbf{u}_{g}$ and $\mathbf{v}$ are odd.

Introduce now the non-negative functions $\Psi, \psi^{\alpha}$ and $\Phi, \varphi^{\alpha}$ which represent the arrival and departure state availability (models can be found in ref. [3]). Moreover, we set

$$
\begin{aligned}
& \Phi_{g}=\Phi\left(N_{g}(\mathbf{k})\right), \Psi_{g}=\Psi\left(N_{g}(\mathbf{k})\right), \\
& \varphi_{\mathbf{P}}^{\alpha}=\varphi^{\alpha}\left(n_{\mathbf{P}}\right), \psi_{\mathbf{P}}^{\alpha}=\psi^{\alpha}\left(n_{\mathbf{P}}\right) .
\end{aligned}
$$

In the non generalized case

$$
\begin{aligned}
& \Psi \alpha\left(N_{g}\right)=1+N_{g}, \Phi\left(N_{g}\right)=N_{g}, \\
& \psi^{\alpha}\left(n_{\mathbf{p}}\right)=1-n_{\mathbf{p}}, \varphi^{\alpha}\left(n_{\mathbf{p}}\right)=n_{\mathbf{p}} .
\end{aligned}
$$

At the right hand sides of the GKTCP equations for phonons [3] we have
Moreover

$$
\left(\frac{\partial N_{g}}{\partial t}\right)_{p \alpha}=2 \int \omega_{p \alpha}\left(\mathbf{P} \rightarrow \mathbf{P}^{\prime}, \mathbf{k}\right)\left(\varphi_{\mathbf{P}}^{\alpha} \psi_{\mathbf{P}^{\prime}}^{\alpha} \Psi_{g}-\psi_{\mathbf{P}}^{\alpha} \varphi_{\mathbf{P}^{\prime}}^{\alpha} \Phi_{g}\right) \frac{\mathrm{d} \mathbf{P}}{8 \pi^{3}},
$$

where $\mathbf{P}^{\prime}=\mathbf{P}-\mathbf{k}+\mathbf{b}\left(\mathbf{P} \rightarrow \mathbf{P}^{\prime}, \mathbf{k}\right)$, is the difference between the number of phonons $\mathbf{k}$ emitted by electrons with 
where

$$
\begin{aligned}
& \mathbf{P}^{\prime}=\mathbf{P}-\mathbf{k}+\mathbf{b}\left(\mathbf{P}^{\prime}, \mathbf{k} \rightarrow \mathbf{P}\right), \\
& \mathbf{P}^{\prime \prime}=\mathbf{P}+\mathbf{k}+\mathbf{b}\left(\mathbf{P}^{\prime \prime} \rightarrow \mathbf{P}, \mathbf{k}\right) .
\end{aligned}
$$

The first term corresponds to to processes with emission of a phonon having quasimomentum $\mathbf{k}$ by an electron having a given quasimomentum $\mathbf{P}$ and reverse processes. The second term corresponds to processes with absorption of a phonon by an electron with quasimomentum $\mathbf{P}$ and reverse processes.

The $\omega$ 's are transition probabilities which account for energy conservation and satisfy the following symmetry relations:

$$
\omega_{p e}\left(\mathbf{P} \rightarrow \mathbf{P}^{\prime}, \mathbf{k}\right)=\omega_{e p}\left(\mathbf{P} \rightarrow \mathbf{P}^{\prime}, \mathbf{k}\right)=\omega_{e p}\left(\mathbf{P}^{\prime}, \mathbf{k} \rightarrow \mathbf{P}\right) .
$$

Moreover, the e-e collision integral reads

$$
\left(\frac{\partial n_{\mathbf{P}}}{\partial t}\right)_{e e}=\iint \omega_{e e}\left(\mathbf{P}, \mathbf{P}_{1} \rightarrow \mathbf{P}^{\prime}, \mathbf{P}_{1}^{\prime}\right)\left(\psi_{\mathbf{P}}^{e} \psi_{\mathbf{P}_{1}} e \varphi_{\mathbf{P}^{\prime}}^{e} \varphi_{\mathbf{P}_{1}^{\prime}}^{e}-\psi_{\mathbf{P}^{\prime}}^{e} \psi_{\mathbf{P}_{1}^{\prime}}^{e} \varphi_{\mathbf{P}}^{e} \varphi_{\mathbf{P}_{1}}^{e}\right) \frac{\mathrm{d} \mathbf{P}_{1} \mathrm{~d} \mathbf{P}_{1}^{\prime}}{64 \pi^{6}},
$$

For holes we have

$$
\left(\frac{\partial n_{\mathbf{P}}}{\partial t}\right)_{h p}=\sum_{g} \int\left[\omega_{h p}\left(\mathbf{P}^{\prime}, \mathbf{k} \rightarrow \mathbf{P}\right)\left(\varphi_{\mathbf{P}^{\prime}}^{h} \psi_{\mathbf{P}}^{h} \Phi_{g}-\psi_{\mathbf{P}^{\prime}}^{h} \varphi_{\mathbf{P}}^{h} \Psi_{g}\right)+\omega_{h p}\left(\mathbf{P}^{\prime \prime} \rightarrow \mathbf{P}, \mathbf{k}\right)\left(\varphi_{\mathbf{P}^{\prime \prime}}^{h} \psi_{\mathbf{P}}^{h} \Psi_{g}-\varphi_{\mathbf{P}}^{h} \psi_{\mathbf{P}^{\prime}}^{h} \Phi_{g}\right)\right] \frac{\mathrm{d} \mathbf{k}}{8 \pi^{3}},
$$

where

$$
\begin{aligned}
& \mathbf{P}^{\prime}=\mathbf{P}-\mathbf{k}+\mathbf{b}\left(\mathbf{P}^{\prime}, \mathbf{k} \rightarrow \mathbf{P}\right), \\
& \mathbf{P}^{\prime \prime}=\mathbf{P}+\mathbf{k}+\mathbf{b}\left(\mathbf{P}^{\prime \prime} \rightarrow \mathbf{P}, \mathbf{k}\right) .
\end{aligned}
$$

The first term corresponds to to processes with emission of a phonon having quasimomentum $\mathbf{k}$ by a hole having a given quasimomentum $\mathbf{P}$ and reverse processes. The second term corresponds to processes with absorp- tion of a phonon by a hole with quasimomentum $\mathbf{P}$ and reverse processes.

The $w$ 's are transition probabilities which account for energy conservation and satisfy the following symmetry relations:

$$
\omega_{p h}\left(\mathbf{P} \rightarrow \mathbf{P}^{\prime}, \mathbf{k}\right)=\omega_{h p}\left(\mathbf{P} \rightarrow \mathbf{P}^{\prime}, \mathbf{k}\right)=\omega_{h p}\left(\mathbf{P}^{\prime}, \mathbf{k} \rightarrow \mathbf{P}\right) .
$$

Moreover, the h-h collision integral reads

$$
\left(\frac{\partial n_{\mathbf{P}}}{\partial t}\right)_{h h}=\iint \omega_{h h}\left(\mathbf{P}, \mathbf{P}_{1} \rightarrow \mathbf{P}^{\prime}, \mathbf{P}_{1}^{\prime}\right)\left(\psi_{\mathbf{P}}^{h} \psi_{\mathbf{P}_{1}}^{h} \varphi_{\mathbf{P}^{\prime}}^{h} \varphi_{\mathbf{P}_{1}^{\prime}}^{h}-\psi_{\mathbf{P}^{\prime}}^{h} \psi_{\mathbf{P}_{1}^{\prime}}^{h} \varphi_{\mathbf{P}}^{h} \varphi_{\mathbf{P}_{1}}^{h}\right) \frac{\mathrm{d} \mathbf{P}_{1} \mathrm{~d} \mathbf{P}_{1}^{\prime}}{64 \pi^{6}},
$$

\section{Asymptotic Expansion and Balance Equations}

By following [1] we expand the kernels and the unknowns with respect to a small parameter $\epsilon$ which takes into account the effect of the umklapp (U) processes in addition to the normal $(\mathrm{N})$ ones (which conserve momentum). We start with carriers (the extension to phonons is trivial). The sought expansions for $n_{\mathbf{p}}^{\alpha}$ and $N_{g}$ read

$$
n_{\mathbf{p}}^{\alpha}=n_{\mathbf{p}}^{N \alpha}+\varepsilon n_{\mathbf{p}}^{U \alpha}, N_{g}=N_{g}^{N}+\varepsilon N_{g}^{U} .
$$

Accordingly

$$
\begin{aligned}
\left(\frac{\partial n_{\mathbf{p}}^{\alpha}}{\partial t}\right)_{\alpha p}= & \left(\frac{\partial n_{\mathbf{p}}^{\alpha}}{\partial t}\right)_{\alpha p}^{N}+\varepsilon\left(\frac{\partial n_{\mathbf{p}}^{\alpha}}{\partial t}\right)_{\alpha p}^{U}+\left(\frac{\partial n_{\mathbf{p}}^{\alpha}}{\partial t}\right)_{\alpha \alpha}^{N} \\
& +\varepsilon\left(\frac{\partial n_{\mathbf{p}}^{\alpha}}{\partial t}\right)_{\alpha \alpha}^{U},
\end{aligned}
$$

where $\omega_{\alpha p}$ and $\omega_{\alpha \alpha}$ read

$$
\omega_{\alpha p}=\omega_{\alpha p}^{N}+\varepsilon \omega_{\alpha p}^{U}, \omega_{\alpha \alpha}=\left(\frac{1}{\varepsilon}\right) \omega_{e e}^{N}+\omega_{e p}^{U} .
$$

Observe that the expansion of $\omega_{e e}$ is singular, since the relaxation due to $e$-e interactions is considered quicker with respect to the case of the $e-p$ ones [6].

We can write now

$$
\left(\frac{\partial n_{\mathbf{p}}^{\alpha}}{\partial t}\right)_{\alpha p}^{N}=\left(\frac{\partial n_{\mathbf{p}}^{\alpha}}{\partial t}\right)_{\alpha p}^{N N}+\varepsilon\left(\frac{\partial n_{\mathbf{p}}^{\alpha}}{\partial t}\right)_{\alpha p}^{N U},
$$

By collecting all these terms and neglecting $\varepsilon^{\ell}$ with $\ell \geq 1$, we have

$$
\begin{aligned}
\mathcal{D}_{\mathbf{p}} n_{\mathbf{p}}^{N \alpha}= & \left(\frac{1}{\varepsilon}\right)\left(\frac{\partial n_{\mathbf{p}}^{\alpha}}{\partial t}\right)_{\alpha \alpha}^{N N}+\left(\frac{\partial n_{\mathbf{p}}^{\alpha}}{\partial t}\right)_{\alpha \alpha}^{N U}+\left(\frac{\partial n_{\mathbf{p}}^{\alpha}}{\partial t}\right)_{\alpha \alpha}^{U N} \\
& +\left(\frac{\partial n_{\mathbf{p}}^{\alpha}}{\partial t}\right)_{\alpha p}^{N N} .
\end{aligned}
$$

At the orders -1 and 0 , we get

$$
\begin{aligned}
& \left(\frac{\partial n_{\mathbf{p}}^{\alpha}}{\partial t}\right)_{\alpha \alpha}^{N N}=0 \\
& \left(\frac{\partial n_{\mathbf{p}}^{\alpha}}{\partial t}\right)_{\alpha \alpha}^{N U}+\left(\frac{\partial n_{\mathbf{p}}^{\alpha}}{\partial t}\right)_{\alpha \alpha}^{U N}+\left(\frac{\partial n_{\mathbf{p}}^{\alpha}}{\partial t}\right)_{\alpha}^{N N}=\mathcal{D}_{\mathbf{p}} n_{\mathbf{p}}^{\alpha N},
\end{aligned}
$$

respectively.

Analogously for phonons

$$
\begin{aligned}
& \left(\frac{\partial N_{g}}{\partial t}\right)_{p p}^{N N}=0 \\
& \left(\frac{\partial N_{g}}{\partial t}\right)_{p p}^{N U}+\left(\frac{\partial N_{g}}{\partial t}\right)_{p p}^{U N}+\sum_{\alpha}\left(\frac{\partial N_{g}}{\partial t}\right)_{p \alpha}^{N N}=\mathcal{D}_{g} N_{g}^{N} .
\end{aligned}
$$


The equations of order -1 for both phonons and electrons are solved (see appendix) by

$$
\ln \left(\frac{\Phi_{g}^{N}}{\Psi_{g}^{N}}\right)=\frac{\left(\mathbf{V}_{p} \cdot \mathbf{k}-\omega_{g}\right)}{T_{p}} \ln \left(\frac{\varphi_{\mathbf{P}}^{N \alpha}}{\psi_{\mathbf{P}}^{N \alpha}}\right)=\frac{\left(\mu_{\alpha}+\mathbf{V}_{\alpha} \cdot \mathbf{p}-\varepsilon_{\mathbf{P}}^{\alpha}\right)}{T_{\alpha}}
$$

where $T_{\alpha}=T_{\alpha}(x, t)$ are the absolute temperatures while $\mu_{\alpha}=\mu_{\alpha}(x, t)$ is the chemical potential of the electron gas (the meaning of $\mathbf{V}_{\alpha}$ is discussed later). Thus, at the leading order of this expansion, we find the drifted (generalized) Bose-Einstein (BE) and Fermi-Dirac (FD) distribution functions. Let us define the functions $\mathcal{B}$ and $\mathcal{F}_{\alpha}$ as follows:

$$
\begin{aligned}
& N_{g}^{N}=\mathcal{B}\left[\mathcal{B}_{p}\left(\omega_{g}-\mathbf{V}_{p} \cdot \mathbf{k}\right)\right], \\
& n_{\mathbf{p}}^{N \alpha}=\mathcal{F}_{\alpha}\left[\mathcal{B}_{\alpha}\left(\varepsilon_{\mathbf{P}}^{\alpha}-\mu_{\alpha}-\mathbf{V}_{\alpha} \cdot \mathbf{p}\right)\right],
\end{aligned}
$$

where $\mathcal{B}_{\alpha}=1 / T_{\alpha}$. In the non-generalized case we have

$$
\mathcal{B}(\zeta)=1 /\left(e^{\zeta}-1\right), \quad \mathcal{F}_{\alpha}(\zeta)=1 /\left(e^{\zeta}+1\right),
$$

that is the BE and FD distribution functions are recovered. Usually [7] $N_{g}^{N}$ and $n_{\mathbf{P}}^{N \alpha}$ are factored into two components, a symmetric component $(0)$ which is even in momentum and an anti-symmetric component (1) which is odd:

$$
\begin{aligned}
N_{g}^{N}=\mathcal{B}\left(\beta_{p} \omega_{g}\right)-\beta_{p} \mathbf{V}_{p} \cdot \mathbf{k} \mathcal{B}^{\prime}\left(\beta \omega_{g}\right)=N_{g}^{0}+N_{g}^{1}, \\
\quad n_{\mathbf{P}}^{N \alpha} \\
=\mathcal{F}\left[\mathcal{B}_{\alpha}\left(\varepsilon_{\mathbf{P}}^{\alpha}-\mu_{\alpha}\right)\right]-\mathcal{B}_{\alpha} \mathbf{V}_{\alpha} \cdot \mathbf{p} \mathcal{F}_{\alpha}^{\prime}\left[\mathcal{B}_{\alpha}\left(\varepsilon_{\mathbf{P}}^{\alpha}-\mu_{\alpha}\right)\right] \\
=n_{\mathbf{P} \alpha}^{0}+n_{\mathbf{P} \alpha}^{1}
\end{aligned}
$$

This simplification is valid when the drift energy is small compared to the thermal energy [5]. Observe that, under this assumption the drift velocities $\langle\mathbf{v}\rangle,\left\langle\mathbf{u}_{g}\right\rangle$ of electrons and phonons are given by respectively by

$$
\left\langle\mathbf{V}_{\alpha}\right\rangle=\frac{\int n_{\mathbf{p}}^{\alpha} \mathbf{v}_{\alpha} \mathrm{d} \mathbf{p}}{\int n_{\mathbf{p}}^{\alpha} l p h a \mathrm{~d} \mathbf{p}}=\mathbf{V}_{\alpha},\left\langle\mathbf{u}_{g}\right\rangle=\frac{\int N_{g} \mathbf{u}_{g} \mathrm{~d} \mathbf{p}}{\int N_{g} \mathrm{~d} \mathbf{p}}=\mathbf{V}_{p}
$$

In fact

$$
\begin{aligned}
\int \mathbf{v}_{\alpha} n_{\mathbf{p}} \mathrm{d} \mathbf{p} & =-\mathcal{B}_{\alpha} \int \mathbf{V}_{\alpha} \cdot \mathbf{p} \mathcal{F}_{\alpha}^{\prime}\left(\mathcal{B}_{\alpha} \varepsilon_{\mathbf{P}}\right) \mathbf{v d} \mathbf{p} \\
& =-\int \mathbf{V}_{\alpha} \cdot \mathbf{p} \frac{\partial}{\partial \mathbf{p}} \mathcal{F}\left(\mathcal{B}_{\alpha} \varepsilon_{\mathbf{p}}^{\alpha}\right) \mathrm{d} \mathbf{p} \\
& =\mathbf{V}_{\alpha} \int \mathcal{F}\left(\mathcal{B}_{\alpha} \varepsilon_{\mathbf{P}}^{\alpha}\right) \mathrm{d} \mathbf{p}
\end{aligned}
$$

and analogously for $\int \mathbf{u}_{g} N_{g} \mathrm{~d} \mathbf{k}$. Moreover, after some calculations we find

$$
\begin{aligned}
& \left(\frac{\partial N_{g}}{\partial t}\right)_{p p}^{N U} \\
= & \beta_{p} \mathbf{V}_{p} \cdot\left\{\int\left[\left(\frac{1}{2}\right) \sum_{g 1 g 2} \Psi_{g}^{0} \Phi_{g 1}^{0} \Phi_{g 2}^{0} \omega_{p p}^{U}\left(\mathbf{k}_{1}, \mathbf{k}_{2} \rightarrow \mathbf{k}\right)\left(\mathbf{k}_{2}+\mathbf{k}_{1}-\mathbf{k}\right)+\sum_{g 1 g 3} \Psi_{g 3}^{0} \Phi_{g}^{0} \Phi_{g 1}^{0} \omega_{p p}^{U}\left(\mathbf{k}, \mathbf{k}_{1} \rightarrow \mathbf{k}_{3}\right)\left(-\mathbf{k}_{3}+\mathbf{k}_{1}+\mathbf{k}\right)\right] \frac{\mathrm{d} \mathbf{k}_{1}}{8 \pi^{3}}\right\} \\
& \left(\frac{\partial n_{\mathbf{P}}}{\partial t}\right)_{\alpha \alpha}^{N U}=\beta_{\alpha} \mathbf{V}_{\alpha} \cdot \iint \omega_{h h}^{U}\left(\mathbf{P}, \mathbf{P}_{1} \rightarrow \mathbf{P}^{\prime}, \mathbf{P}_{1}^{\prime}\right) \psi_{\mathbf{P}^{\prime}}^{0 \alpha} \psi_{\mathbf{P}_{1}^{\prime}}^{0 \alpha} \varphi_{\mathbf{P}}^{0 \alpha} \varphi_{\mathbf{P}_{1}}^{0}\left(\mathbf{P}+\mathbf{P}_{1}-\mathbf{P}^{\prime}-\mathbf{P}_{1}^{\prime}\right) \frac{\mathrm{d} \mathbf{P}_{1} \mathrm{~d} \mathbf{P}_{1}^{\prime}}{64 \pi^{6}}
\end{aligned}
$$

Finally

$$
\begin{aligned}
& \left(\frac{\partial N_{g}}{\partial t}\right)_{p \alpha}^{N N} \\
= & 4 \sum_{g} \int \omega_{p \alpha}\left(\mathbf{P}^{\prime}, \mathbf{k} \rightarrow \mathbf{P}\right)\left(\psi_{\alpha} \varphi_{\alpha}^{\prime} \Phi_{g} \varphi_{\alpha} \psi_{\alpha}^{\prime} \Psi_{g}\right)^{1 / 2} \\
& \times \sinh \left\{\left[\left(\beta_{\alpha} \mathbf{V}_{\alpha}-\beta_{p} \mathbf{V}_{p}\right) \cdot \mathbf{k}+\left(\beta_{p}-\beta_{\alpha}\right) \omega_{g}\right] / 2\right\} \frac{\mathrm{d} \mathbf{P}}{8 \pi^{3}}
\end{aligned}
$$

and, in the linear non-equilibrium thermodynamic approach,

$$
\begin{aligned}
\left(\frac{\partial N_{g}}{\partial t}\right)_{p \alpha}^{N N}= & 2 \sum_{g} \int \omega_{p \alpha}\left(\mathbf{P}^{\prime}, \mathbf{k} \rightarrow \mathbf{P}\right) \psi_{\alpha}^{*} \varphi_{\alpha}^{\prime *} \Phi_{g}^{*} \\
& \times\left[\left(\beta_{e} \mathbf{V}_{e}-\beta_{p} \mathbf{V}_{p}\right) \cdot \mathbf{k}+\left(\beta_{p}-\beta_{e}\right) \omega_{g}\right] \frac{\mathrm{d} \mathbf{P}}{8 \pi^{3}},
\end{aligned}
$$

where $*$ stands for equilibrium, that is $\mathbf{V}_{h}=\mathbf{V}_{e}=\mathbf{V}_{p}(=0$ in the present approximation) and $\beta_{h}=\beta_{e}=\beta_{p}$ (see Appendix).

The equations of order 0 are the starting point of our macroscopic model. By projecting the electron one over 1 the continuity equation for electrons and holes read

$$
\frac{\partial}{\partial t} \int n_{\mathbf{p} \alpha}^{0}+\nabla \cdot \int \mathbf{v}^{\alpha} n_{p \alpha}^{1} \mathrm{~d} \mathbf{p}=0
$$

By projecting the electron equation over $\mathbf{p}$ and the phonon ones on $\mathbf{k}$ we get the following balance equations for momentum $\left(e_{\alpha}=e,-e, \alpha=e, h\right)$ :

$$
\begin{aligned}
& \frac{\partial}{\partial t} \int n_{p \alpha}^{1} \mathbf{p d} \mathbf{p}+\nabla \cdot \int n_{\mathbf{p} \alpha}^{0} \mathbf{v} \otimes \mathbf{p d} \mathbf{p} \\
= & -e_{\alpha} \mathbf{E} \int n_{\mathbf{p}}^{0} \mathrm{~d} \mathbf{p}+\int\left(\frac{\partial n_{\mathbf{p}}^{\alpha}}{\partial t}\right)_{\alpha p}^{N N} \mathbf{p} \mathrm{d} \mathbf{p}+\int\left(\frac{\partial n_{\mathbf{p}}^{\alpha}}{\partial t}\right)_{\alpha \alpha}^{N U} \mathbf{p d} \mathbf{p} \\
& \frac{\partial}{\partial t} \int N_{g}^{1} \mathbf{k d} \mathbf{k}+\nabla \cdot \sum_{g} \int N_{g}^{0} \mathbf{u}_{g} \otimes \mathbf{k d} \mathbf{k} \\
= & +\int\left[\sum_{g}\left(\frac{\partial N_{g}}{\partial t}\right)_{p p}^{N U}+\sum_{\alpha}\left(\frac{\partial N_{g}}{\partial t}\right)_{p \alpha}^{N U}\right] \mathbf{k d k},
\end{aligned}
$$


where we took advantage of

$$
\int\left(\frac{\partial n_{\mathbf{p}}^{\alpha}}{\partial t}\right)_{p p}^{U N} \mathbf{p} \mathrm{d} \mathbf{p}=0, \quad \sum_{g} \int\left(\frac{\partial N_{g}}{\partial t}\right)_{p p}^{U N} \mathbf{k} \mathrm{d} \mathbf{k}=0,
$$

due to momentum conservation for N-processes For the same reason we have

$$
2 \int\left(\frac{\partial n_{\mathbf{p}}^{\alpha}}{\partial t}\right)_{p \alpha}^{N N} \mathbf{p d} \mathbf{p}+\sum_{g} \int\left(\frac{\partial N_{g}}{\partial t}\right)_{p \alpha}^{N N} \mathbf{k d} \mathbf{k}=0,
$$

where

$$
\sum_{g} \int\left(\frac{\partial N_{g}}{\partial t}\right)_{p \alpha}^{N N} \mathbf{k d} \mathbf{k}=\left(\beta_{\alpha} \mathbf{V}_{\alpha}-\beta_{p} \mathbf{V}_{p}\right) \cdot \sum_{g} \iint 2 \omega_{p e}^{N}\left(\mathbf{P}^{\prime}, \mathbf{k} \rightarrow \mathbf{P}\right) \psi^{*} \varphi^{\prime *} \Phi_{g}^{*} \mathbf{k} \otimes \mathbf{k d} \mathbf{p d} \mathbf{k} / 8 \pi^{3}
$$

This term is responsible of momentum relaxation.

Finally, by projecting the electron equation over $\varepsilon_{\mathbf{P}}$,

$$
\begin{aligned}
& \sum_{g} \frac{\partial}{\partial t} \int \varepsilon_{\mathbf{p}}^{\alpha} n_{p}^{0} \mathrm{~d} \mathbf{p}+\nabla \cdot \int \mathbf{v}^{\alpha} \varepsilon_{\mathbf{p}}^{\alpha} n_{p}^{1} \mathrm{~d} \mathbf{p} \\
= & -2 \sum_{g} \mathbf{E} \cdot \int \mathbf{v} n_{p}^{\alpha} \mathrm{d} \mathbf{p}+\sum_{g} \int\left(\frac{\partial n_{\mathbf{p}}}{\partial t}\right)_{\alpha p}^{N N} \varepsilon_{\mathbf{p}} \mathrm{d} \mathbf{p}+\int\left(\frac{\partial n_{\mathbf{p}}}{\partial t}\right)_{\alpha \alpha}^{N U} \varepsilon_{\mathbf{p}} \mathrm{d} \mathbf{p} \frac{\partial}{\partial t} \sum_{g} \int \omega_{g} N_{g}^{0} \mathrm{~d} \mathbf{k}+\nabla \cdot \sum_{g} \int \mathbf{u}_{g} N_{g}^{1} \mathrm{~d} \mathbf{k} \\
= & +\int \sum_{g}\left(\frac{\partial N_{g}}{\partial t}\right)_{p e}^{N N} \omega_{g} \mathrm{~d} \mathbf{k}+\sum_{g} \int\left(\frac{\partial N_{g}}{\partial t}\right)_{e e}^{N U} \omega_{g} \mathrm{~d} \mathbf{k},
\end{aligned}
$$

where we took advantage of

$$
\int\left(\frac{\partial n_{\mathbf{p}}}{\partial t}\right)_{\alpha \alpha}^{U N} \varepsilon_{\mathbf{p}} \mathrm{d} \mathbf{p}=0, \sum_{g} \int\left(\frac{\partial N_{g}}{\partial t}\right)_{p p}^{U N} \omega_{g} \mathrm{~d} \mathbf{k}=0
$$

due to energy conservation for $\mathrm{N}$-processes. For the same

$$
\int\left(\frac{\partial n_{\mathbf{p}}}{\partial t}\right)_{p \alpha}^{N N} \omega_{g} \mathrm{~d} \mathbf{k}=2\left(\beta_{p}-\beta_{\alpha}\right) \sum_{g} \iint \omega_{p \alpha}\left(\mathbf{P}^{\prime}, \mathbf{k} \rightarrow \mathbf{P}\right) \psi^{*} \varphi^{\prime *} \Phi_{g}^{*} \omega_{g}^{2} \mathrm{~d} \mathbf{p d} \mathbf{k} / 8 \pi^{3} .
$$

This term, responsible of the thermal relaxation, generalizes the results which can be found in the literature [6], by means of the functions "phi" and "psi" we introduced.

Equations (4)-(6) constitute now a closed set of equations for the unknowns $\mu, \beta_{\alpha}, \mathbf{V}_{\alpha}(\alpha=e, p)$ which recall the extended thermodynamical one [4].

\section{Revised Drift-Diffusion Approximation}

In the drift-diffusion approximation we assume that the total momentum of the mixture does not vary appreciably reason we have

$$
2 \int\left(\frac{\partial n_{\mathbf{p}}}{\partial t}\right)_{p \alpha}^{N N} \varepsilon_{\mathbf{p}} \mathrm{d} \mathbf{p}+\sum_{g} \int\left(\frac{\partial N_{g}}{\partial t}\right)_{p \alpha}^{U N} \omega_{g} \mathrm{~d} \mathbf{k}=0,
$$

where

$$
\begin{aligned}
& B_{p}=-\frac{1}{2} \sum_{g 1 g 2 g 3} \iint \Phi_{g 2}^{0} \Phi_{g 3}^{0} \Psi_{g 1}^{0} \omega_{p p}\left(\mathbf{k}_{2}, \mathbf{k}_{3} \rightarrow \mathbf{k}_{1}\right)\left(\mathbf{k}_{1}-\mathbf{k}_{2}-\mathbf{k}_{3}\right) \otimes\left(\mathbf{k}_{1}-\mathbf{k}_{2}-\mathbf{k}_{3}\right) \frac{\mathrm{d} \mathbf{k}_{1} \mathrm{~d} \mathbf{k}_{2}}{8 \pi^{3}} \\
& B_{e}=-\left(\frac{1}{4}\right) \iiint \omega_{e e}\left(\mathbf{p}, \mathbf{p}_{1} \rightarrow \mathbf{p}^{\prime}, \mathbf{p}_{1}^{\prime}\right)\left(\mathbf{p}+\mathbf{p}_{1}-\mathbf{p}^{\prime}-\mathbf{p}_{1}^{\prime}\right) \otimes\left(\mathbf{p}+\mathbf{p}_{1}-\mathbf{p}^{\prime}-\mathbf{p}_{1}^{\prime}\right) \frac{\mathrm{d} \mathbf{p d} \mathbf{p}_{1} \mathrm{~d} \mathbf{p}^{\prime}}{64 \pi^{6}}
\end{aligned}
$$

and

$$
D_{\alpha}=2 \sum_{g} \iint \omega_{p \alpha}\left(\mathbf{p}^{\prime}, \mathbf{k} \rightarrow \mathbf{p}\right) \psi_{\varepsilon}^{*} \varphi_{\alpha}^{\prime *} \Phi_{g}^{*} \mathbf{k} \otimes \mathbf{k d} \mathbf{p d} \mathbf{k} / 8 \pi^{3} .
$$

Moreover

$$
\mathbf{X}_{e}=-2 \beta_{e}^{2} R_{2} \cdot \nabla T_{e}-2 \beta_{e} R_{1} \cdot \mathbf{E}_{e},
$$

over the momentum relaxation time [7]. Then the momentum balance equations read

$$
\begin{aligned}
& D_{e} \cdot\left(\hat{\mathbf{V}}_{p}-\hat{\mathbf{V}}_{e}\right)+B_{e} \cdot \hat{\mathbf{V}}_{e}=\mathbf{X}_{e} \\
& D_{h} \cdot\left(\hat{\mathbf{V}}_{p}-\hat{\mathbf{V}}_{h}\right)+B_{h} \cdot \hat{\mathbf{V}}_{h}=\mathbf{X}_{h} \\
& D_{e} \cdot\left(\hat{\mathbf{V}}_{e}-\hat{\mathbf{V}}_{p}\right)+D_{h} \cdot\left(\hat{\mathbf{V}}_{h}-\hat{\mathbf{V}}_{p}\right)+B_{p} \cdot \mathbf{V}_{p}=\mathbf{X}_{p}
\end{aligned}
$$

The tensors $B_{\alpha}$, can be written in the following symmetric form

$$
\begin{aligned}
& \mathbf{X}_{h}=-2 \beta_{h}^{2} R_{2} \cdot \nabla T_{h}+2 \beta_{h} e R_{4} \cdot \mathbf{E}_{h}, \\
& \mathbf{X}_{p}=-\beta_{p} R_{5} \cdot \nabla T_{p},
\end{aligned}
$$

where $\mathbf{E}_{e}=\mathbf{E}+(1 / e) \nabla \mu_{e}, \mathbf{E}_{h}=\mathbf{E}-(1 / e) \nabla \mu_{h}$, , and

$$
R_{\ell}=\int \mathcal{F}^{\prime}\left[\beta_{\alpha}\left(\varepsilon_{\mathbf{p}}^{\alpha}-\mu_{\alpha}\right)\right] \mathbf{p} \otimes \mathbf{v d p} \quad \alpha=e, h \quad \ell=1,3
$$




$$
\begin{gathered}
R_{\ell}=\int \mathcal{F}^{\prime}\left[\beta_{\alpha}\left(\varepsilon_{\mathbf{P}}^{\alpha}-\mu\right)\right]\left(\varepsilon_{\mathbf{P}}^{\alpha}-\mu_{\alpha}\right) \mathbf{p} \otimes \mathbf{v d} \mathbf{p} \\
\alpha=e, h \quad \ell=2,4 \\
R_{3}=\sum_{g} \int \mathcal{B}^{\prime}\left(\beta_{p} \omega_{g}\right) \omega_{g} \mathbf{k} \otimes \mathbf{u}_{g} \mathrm{~d} \mathbf{k},
\end{gathered}
$$

where we can write

$$
R_{\ell}=r_{\ell} I \quad \ell=1,3
$$

Since in the present approximation all the moments are calculated by means of drifted (generalized) FD or BE distribution functions, the electrical $\left(\mathbf{J}_{e}\right)$ and thermal $\left(\mathbf{u}_{e}\right.$, $\mathbf{u}_{p}$ ) currents are given by [8]

$$
\begin{aligned}
\mathbf{J}_{e}=-\frac{e}{4 \pi^{3}} \int \mathbf{v}_{e} n_{p}^{e} \mathrm{~d} \mathbf{p} & =\frac{e}{4 \pi^{3}} \int \mathbf{v}_{e} \hat{\mathbf{V}}_{e} \cdot \mathbf{p} \mathcal{F}^{\prime}\left[\beta\left(\varepsilon_{\mathbf{p}}^{\alpha}-\mu_{e}\right)\right] \mathrm{d} \mathbf{p} \quad \text { By introducing the } \hat{\mathbf{V}}_{\alpha} \quad \text { (see Append } \\
\mathbf{J}_{e} & =-\frac{e}{4 \pi^{3}}\left(2 \beta_{e}\left(\mathrm{~K}_{11} \cdot \mathbf{E}_{e}-\mathrm{K}_{13} \cdot \mathbf{E}_{h}\right)+2 \beta_{e}^{2} \mathrm{~K}_{12} \cdot \nabla T_{e}+2 \beta_{h}^{2} \mathrm{~K}_{14} \cdot \nabla T_{h}+\beta_{p}^{2} \mathrm{~K}_{15} \cdot \nabla T_{p}\right) \\
\mathbf{U}_{e} & =\frac{\beta}{4 \pi^{3}}\left(2 \beta_{e}\left(\mathrm{~K}_{21} \cdot \mathbf{E}_{e}-\mathrm{K}_{23} \cdot \mathbf{E}_{h}\right)+2 \beta_{e}^{2} \mathrm{~K}_{22} \cdot \nabla T_{e}+2 \beta_{h}^{2} \mathrm{~K}_{24} \cdot \nabla T_{h}+\beta_{p}^{2} \mathrm{~K}_{25} \cdot \nabla T_{p}\right) \\
\mathbf{J}_{h} & =\frac{e}{4 \pi^{3}}\left(2 \beta_{e}\left(\mathrm{~K}_{31} \cdot \mathbf{E}_{e}-\mathrm{K}_{33} \cdot \mathbf{E}_{h}\right)+2 \beta_{e}^{2} \mathrm{~K}_{32} \cdot \nabla T_{e}+2 \beta_{h}^{2} \mathrm{~K}_{14} \cdot \nabla T_{h}+\beta_{p}^{2} \mathrm{~K}_{35} \cdot \nabla T_{p}\right) \\
\mathbf{U}_{h} & =\frac{\beta}{4 \pi^{3}}\left(2 \beta_{e}\left(\mathrm{~K}_{41} \cdot \mathbf{E}_{e}-\mathrm{K}_{43} \cdot \mathbf{E}_{h}\right)+2 \beta_{e}^{2} \mathrm{~K}_{42} \cdot \nabla T_{e}+2 \beta_{h}^{2} \mathrm{~K}_{44} \cdot \nabla T_{h}+\beta_{p}^{2} \mathrm{~K}_{45} \cdot \nabla T_{p}\right) \\
\mathbf{U}_{p} & =\frac{1}{4 \pi^{3}}\left(2 \beta_{e}\left(\mathrm{~K}_{51} \cdot \mathbf{E}_{e}-\mathrm{K}_{53} \cdot \mathbf{E}_{h}\right)+2 \beta_{e}^{2} \mathrm{~K}_{52} \cdot \nabla T_{e}+2 \beta_{h}^{2} \mathrm{~K}_{54} \cdot \nabla T_{h}+\beta_{p}^{2} \mathrm{~K}_{55} \cdot \nabla T_{p}\right)
\end{aligned}
$$

$$
\begin{aligned}
\mathbf{J}_{h} & =\frac{e}{4 \pi^{3}} \int \mathbf{v}_{e} n_{p}^{h} \mathrm{~d} \mathbf{p}=\frac{e}{4 \pi^{3}} \int \mathbf{v}_{h} \hat{\mathbf{V}}_{h} \cdot \mathbf{p} \mathcal{F}^{\prime}\left[\beta\left(\varepsilon_{\mathbf{P}}^{\alpha}-\mu_{h}\right)\right] \mathrm{d} \mathbf{p} \\
\mathbf{U}_{\alpha} & =\frac{1}{4 \pi^{3}} \int \mathbf{v}_{\alpha}\left(\varepsilon_{\mathbf{P}}^{\alpha}-\mu_{\alpha}\right) n_{p}^{e} \mathrm{~d} \mathbf{p} \\
& =-\frac{1}{4 \pi^{3}} \int \mathbf{v}_{\alpha} \hat{\mathbf{V}}_{\alpha} \cdot \mathbf{p} \mathcal{F}^{\prime}\left[\beta_{\alpha}\left(\varepsilon_{\mathbf{P}}^{\alpha}-\mu_{\alpha}\right)\right]\left(\varepsilon_{\mathbf{P}}^{\alpha}-\mu_{\alpha}\right) \mathrm{d} \mathbf{p} \\
\alpha & =e, h \\
\mathbf{U}_{g} & =\frac{1}{8 \pi^{3}} \sum_{g} \int \omega_{g} \mathbf{u}_{g} N_{g} \mathrm{~d} \mathbf{k} \\
& =-\frac{1}{8 \pi^{3}} \sum_{g} \int \omega_{g} \mathbf{u}_{g} \hat{\mathbf{V}}_{p} \cdot \mathbf{k} \mathcal{B}^{\prime}\left(\beta \omega_{g}\right) \mathrm{d} \mathbf{k}
\end{aligned}
$$

By introducing the $\hat{\mathbf{V}}_{\alpha}$ (see Appendix 2) we obtain

A further step, for future work, could be the introduction of holes and photons in the model, in order to account fo generation-recombination effects.

$$
\mathrm{K}_{\ell m}=\widetilde{\mathrm{K}}_{m \ell}
$$

And, in the presence of and external applied field

$$
\mathrm{K}_{\ell m}(-B)=\widetilde{\mathrm{K}}_{m \ell}(B)
$$

since

$$
R_{\ell}=r_{\ell} I \quad \ell=1,3
$$

\section{Conclusions}

A new two-fluid model for electron-phonon system has been proposed, which is certainly related to the extended thermodynamical one [4], for the purpose of calculating energy and momentum relaxation rates which generalize the results available in the literature [6]. The treatment resorts here strictly to kinetic theory, so that the model is closed. This means that we do not need adjustment of some free parameters (namely the relaxation times) by means of comparisons with Monte Carlo calculations. A revised drift-diffusion approximation has been derived. An obvious improvement with respect to the classical drift-diffusion model is constituted by the introduction of an energy balance equation. The fulfillment of the symmetry Onsager relations is not trivial, since it cannot taken for granted in many macroscopic models.

\section{REFERENCES}

[1] A. Rossani and A. M. Scarfone, "Generalized Kinetic Theory of Electrons and Phonons: Models, Equilibrium, Stability," Physica B: Condensed Matter, Vol. 334, 2003, pp. 292-297. doi:10.1016/S0921-4526(03)00079-6

[2] I. Koponen, "Thermalization of an Electron-Phonon System in a Non-Equilibrium Statecharacterized by Fractal Distribution of Phonon Excitations," Physical Review E, Vol. 55, No. 6, 1997, pp. 7759-7762.

[3] A. Rossani, "Generalized Kinetic Theory of Electrons and Phonons," Physica A: Statistical Mechanics and Its Applications, Vol. 305, No. 1-2, 2002, pp. 323-329. doi:10.1016/S0378-4371(01)00682-3

[4] A. M. Anile and S. Pennisi, "Thermodynamic Derivation of the Hydrodynamical Model for Charge Transport in Semiconductors," Physical Review B, Vol. 46, No. 20, 1992, pp. 13186-13193.

[5] A. Rossani, G. Spiga and A. Domaingo, "Band-Trap Capture and Emission in the Generalized Kinetic Theory of Electrons and Holes," Journal of Physics A: Mathematical and General, Vol. 36, No. 48, 2003, Article ID: 11955. doi:10.1088/0305-4470/36/48/004

[6] N. B. Abdallah, P. Degond and S. Genyeis, "An Energy- 
Transport Model for Semiconductors Derived from the Boltzmann Equation," Journal of Statistical Physicss, Vol. 84, No. 1-2, 1996, pp. 205-231. doi:10.1007/BF02179583

[7] A. Rossani and G. Spiga, "Auger Effect in the Generalized Kinetic Theory of Electrons and Holes," Journal of

\section{Appendix 1}

Consider the equations at order -1 :

$$
\left(\frac{\partial N_{g}}{\partial t}\right)_{p p}^{N N}=0, \quad\left(\frac{\partial n_{\mathbf{p}}}{\partial t}\right)_{e e}^{N N}=0, \quad\left(\frac{\partial n_{\mathbf{p}}}{\partial t}\right)_{p p}^{N N}=0 .
$$

By means of the usual methods of kinetic theory it can be shown that these conditions are equivalent to

$$
\begin{gathered}
\Phi_{g}^{N} \Phi_{g 1}^{N} \Phi_{g 2}^{N}=\Phi_{g}^{N} \Phi_{g 1}^{N} \Psi_{g 2}^{N} \quad \forall \mathbf{k}, \mathbf{k}_{1} \\
\psi_{\mathbf{p}}^{\alpha} \psi_{\mathbf{p}_{1}}^{\alpha} \varphi_{\mathbf{p}^{\prime}}^{\alpha} \varphi_{\mathbf{p}_{1}^{\prime}}^{\alpha}=\psi_{\mathbf{p}^{\prime}}^{\alpha} \psi_{\mathbf{p}_{1}^{\prime}}^{\alpha} \varphi_{\mathbf{p}}^{\alpha} \varphi_{\mathbf{p}_{1}}^{\alpha} \forall \mathbf{p}, \mathbf{p}_{1} .
\end{gathered}
$$

Condition (A1) shows that $\ln \left(\Phi_{g}^{N} / \Psi_{g}^{N}\right)$ is a collisional invariant for phonons. In the case of $\mathrm{N}$-processes

$$
\ln \left(\Phi_{g}^{N} / \Psi_{g}^{N}\right)=\left(\mathbf{V}_{p} \cdot \mathbf{k}-\omega_{g}\right) / T_{p}
$$

Condition (A2) shows that $\ln \left(\varphi_{\mathbf{p}}^{\alpha N} / \psi_{\mathbf{p}}^{\alpha N}\right)$ is a collisional invariant for electrons. In the case of $\mathrm{N}$-processes

$$
\ln \left(\varphi_{\mathbf{p}}^{N} / \psi_{\mathbf{p}}^{N}\right)=\left(\mathbf{V}_{e} \cdot \mathbf{k}-\varepsilon_{\mathbf{p}}+\mu\right) / T_{e} .
$$

On the other hand the equilibrium condition for ep interactions reads

$$
\varphi_{\mathbf{p}}^{N} \psi_{\mathbf{p}^{\prime}}^{N} \Psi_{g}^{N}=\varphi_{\mathbf{p}^{\prime}}^{N} \psi_{\mathbf{p}}^{N} \Phi_{g}^{N} \quad \forall \mathbf{p}, \mathbf{k}
$$

which give

$$
\left(\beta_{\alpha} \mathbf{V}_{\alpha}-\beta_{p} \mathbf{V}_{p}\right) \cdot \mathbf{k}+\omega_{g}\left(\beta_{p}-\beta_{\alpha}\right)=0
$$

or, since $\omega_{g}$ is even

$$
\begin{gathered}
-\left(\beta_{\alpha} \mathbf{V}_{\alpha}-\beta_{p} \mathbf{V}_{p}\right) \cdot \mathbf{k}+\omega_{g}\left(\beta_{p}-\beta_{\alpha}\right)=0 \\
\mathbf{V}_{e}=\mathbf{V}_{h}=\mathbf{V}_{p}, \quad \beta_{e}=\beta_{h}=\beta_{p} .
\end{gathered}
$$

Mathematical Physics, Vol. 47, No. 13, 2006, Article ID: 013301. doi:10.1063/1.2161020

[8] N. B. Abdallah and P. Degond, "On a Hierarchy of Macroscopic Models for Semiconductors," Journal of Mathematical Physics, Vol. 37, No. 7, 1996, pp. 3306-3333. doi: $10.1063 / 1.531567$

\section{Appendix 2}

Rewrite the equations as

$$
\begin{aligned}
& \mathbf{A}_{e e} \cdot \hat{\mathbf{V}}+\mathbf{A}_{e p} \cdot \hat{\mathbf{V}}_{p}=\mathbf{X}_{e} \\
& \mathbf{A}_{p e} \cdot \hat{\mathbf{V}}_{e}+\mathbf{A}_{p h} \cdot \hat{\mathbf{V}}_{h}+\mathbf{A}_{p p} \cdot \hat{\mathbf{V}}_{p}=\mathbf{X}_{p} \\
& \mathbf{A}_{h h} \cdot \hat{\mathbf{V}}_{h}+\mathbf{A}_{h p} \cdot \hat{\mathbf{V}}_{p}=\mathbf{X}_{h},
\end{aligned}
$$

which give

$$
\begin{aligned}
& \hat{\mathbf{V}}_{e}=\mathbf{A}_{e e}^{-1} \cdot\left(\mathbf{X}_{\ell}-\mathbf{A}_{e p} \cdot \hat{\mathbf{V}}_{p}\right) \\
& \hat{\mathbf{V}}_{h}=\mathbf{A}_{h h}^{-1} \cdot\left(\mathbf{X}_{h}-\mathbf{A}_{h p} \cdot \hat{\mathbf{V}}_{p}\right)
\end{aligned}
$$

and

$$
\begin{aligned}
& \mathbf{A}_{p e} \cdot \mathbf{A}_{e e}^{-1} \cdot\left(\mathbf{X}_{\ell}-\mathbf{A}_{e p} \cdot \hat{\mathbf{V}}_{p}\right)+\mathbf{A}_{p h} \cdot \mathbf{A}_{h h}^{-1} \cdot\left(\mathbf{X}_{h}-\mathbf{A}_{h p} \cdot \hat{\mathbf{V}}_{p}\right) \\
& +\mathbf{A}_{p p} \cdot \hat{\mathbf{V}}=\mathbf{X}_{p} .
\end{aligned}
$$

so that we get finally the following Onsager symmetry relations:

$$
\mathrm{K}_{\ell m}=\widetilde{\mathrm{K}}_{m \ell} .
$$

And, in the presence of and external applied field

$$
\mathrm{K}_{\ell m}(-B)=\widetilde{\mathrm{K}}_{m \ell}(B)
$$

since

$$
R_{\ell}=r_{\ell} I \quad \ell=1,3 \text {. }
$$

